\title{
Primary breast angiosarcoma: a case report
}

\author{
Fui Tin Pang ${ }^{1,2^{*}}$, Sze Yuen Lee ${ }^{1}$ and Maninderpal Kaur ${ }^{1}$
}

\begin{abstract}
Background: Angiosarcomas are rare and fast growing tumors that arise from vascular structures. This case report is significant because it is rare and may cause treatment delay if not considered as a differential diagnosis. Hence, we aim to inform efficient, appropriate, and evidence-based imaging practice to the benefit of patients regionally and worldwide.

Case presentation: Thirty-five-year-old lady, presented with a large left breast swelling. She had recent history of trauma to the breast which initially caused confusion regarding the possible diagnosis. Ultrasound and MRI raised high suspicion of an angiosarcoma. Patient went through embolectomy prior to mastectomy and axillary clearance. Histopathology confirmed the diagnosis of an angiosarcoma. She had no distant metastases at the time of diagnosis and the patient was treated with chemotherapy and radiotherapy. However, she returned with recurrence within 1-year duration.

Conclusion: Primary breast angiosarcomas are rare aggressive vascular tumors with generally poor prognosis and no pathognomonic signs on radiological imaging, especially mammogram. Ultrasound and MRI do help in characterizing these tumors and raising the suspicion of a vascular tumor. Ultrasound-guided biopsy is recommended to avoid unnecessary complications. In young patients with rapidly growing, highly vascular breast tumors, a differential diagnosis of primary angiosarcomas should be considered until proven otherwise.
\end{abstract}

Keywords: Primary angiosarcomas, Breast, Fast growing tumor

\section{Background}

Angiosarcomas are aggressive tumors that arise from vascular structures and often have metastases at the time of diagnosis. They are rare and account for $<1 \%$ of all sarcomas $[1$, 2]. They can occur in the breast and be further categorized into primary and secondary breast angiosarcomas.

Primary breast angiosarcomas are exceedingly rare as compared to secondary breast angiosarcomas, representing $0.04 \%$ of all malignant breast tumors [3, 4]. They commonly occur in younger age groups, whereas secondary breast angiosarcomas occur in older age groups and are strongly related to post-radiation therapy or prolonged lymphedema secondary to mastectomy and axillary clearance.

* Correspondence: pangfuitin@gmail.com

${ }^{1}$ Hospital Tengku Ampuan Rahimah, Klang, Malaysia

${ }^{2}$ Saint Joseph's Health Care London, London, Canada
Here, we present a case of primary breast angiosarcoma, correlating the clinical presentation, imaging, and pathological findings.

\section{Case presentation}

A 35-year-old lady, single, and nulliparous, presented to our hospital in October 2017 with painful left breast swelling. She had no past medical illnesses nor family history of breast or ovarian cancer. Initial history taken by the clinician stated that the patient had a history of fall 2 weeks prior in the bathroom, hitting her breast against the toilet bowl and subsequently developed progressive left breast swelling.

On physical examination, the left breast was grossly swollen, firm with bluish discoloration of the overlying skin. There were no palpable left axillary lymph nodes. Bruises were seen on other parts of the body. With the given history and clinical findings, hematomas were first suspected by the primary team. However, a repeated 


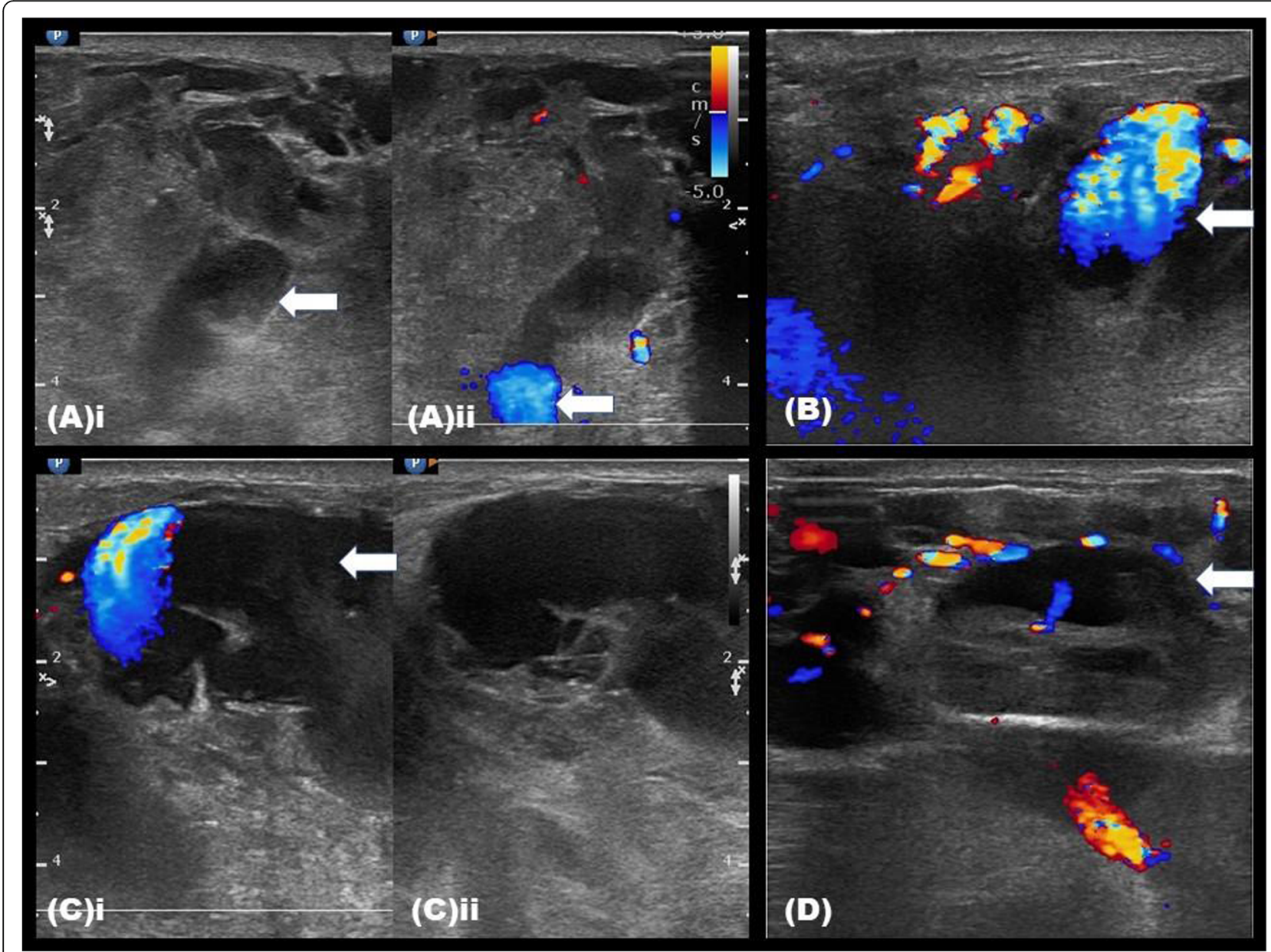

Fig. 1 a-d Ultrasound showed a large irregular mixed echogenic mass with solid and cystic components within seen occupying almost the entire breast. Multiple large vessels with turbulent flow and pulsations were seen within this mass (arrows)

sonography showed a large irregular mixed echogenic mass with solid and cystic components within, occupying almost the entire left breast (Fig. 1). Multiple large vessels with turbulent flow and pulsations were seen within this mass. There were no significant enlarged left axillary nodes. Right breast was normal. Mammogram was not performed as the patient was in great pain. When the patient was prompted with more specific questions, she stated that she had actually felt a lump in her left breast three months prior to the fall.

The primary team attempted core biopsy of the left breast and encountered difficulty in securing hemostasis post-biopsy. The pathology specimen demonstrated blood clots and was therefore inconclusive.

Magnetic resonance imaging (MRI) of the breast revealed a large high $\mathrm{T} 2$ signal intensity mass occupying the entire left breast with blooming artifacts within. It showed rapid enhancement post-contrast administration with progressive and prolonged enhancement in the delayed phase. There were no abnormalities in the right breast or both axillary regions (Fig. 2). Given the clinical history, ultrasound, and MRI findings, an aggressive vascular tumorlike angiosarcoma was highly likely in this case.

She was then referred to Hospital Kuala Lumpur for embolectomy followed by left mastectomy and axillary clearance on 8th November 2017. Pathologically, angiosarcoma was confirmed. It measured $10.0 \times 10.5 \times 7.0$ $\mathrm{cm}$ on its largest axis. All margins were reported clear. There was no overlying skin or underlying muscle invasion. All 17 lymph nodes that were excised showed no malignancy. The histopathological sections from the solitary tumor showed multifocal, ill-defined vascular tumor consisting of proliferating and anastomosing vascular channels, dissecting through the adipose tissue and lobular stroma. The neoplastic vascular channels were lined by atypical endothelial cells characterized by moderate to severe nuclear pleomorphism, hyperchromatic nuclei, and moderate amount of cytoplasm. Immunohistochemistry studies showed the neoplastic cells were positive for CD31 and CD34 but negative for CKAE1/ 


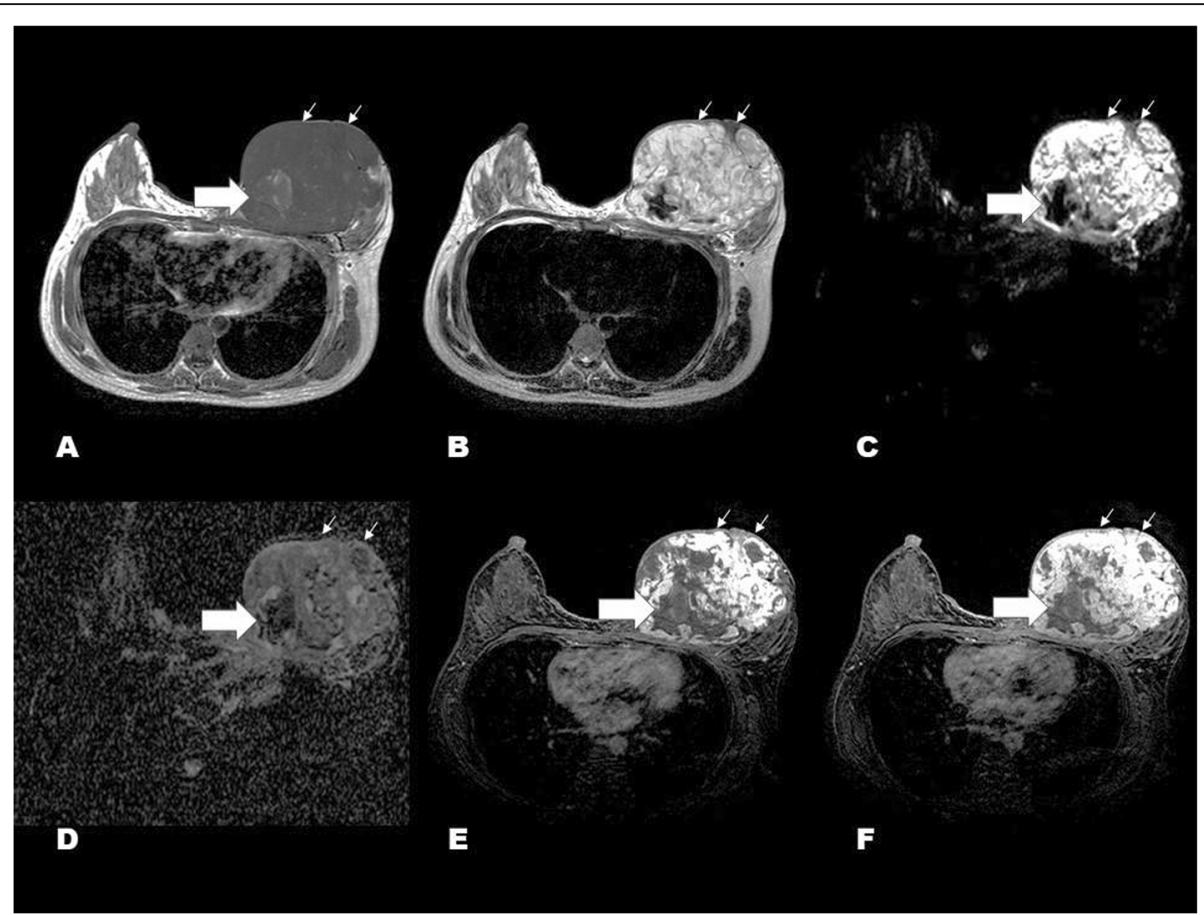

Fig. $2 \mathrm{MRl}$ of the breast revealed a large low T1 (a), high T2 (b) signal intensity mass (thin arrow) occupying the entire left breast with 'blood lake' (thick arrow) within. It demonstrated restricted diffusion on DWI and ADC (c, d), rapid enhancement post-contrast administration with progressive and prolonged enhancement in the delayed phase $(\mathbf{e}, \mathbf{f})$

AE3. Computed tomography (CT) scan showed no evidence of distant metastasis. The patient then underwent 6 cycles of chemotherapy followed by adjuvant radiotherapy. Patient was discharged well. However, she returned with recurrence within 1-year duration.

\section{Discussion}

Primary breast angiosarcomas are rare with an incidence rate of $0.04 \%$ of all malignant breast tumors [3-5]. They have no known risk factors. Some articles mentioned that they might be associated with pregnancy while others stated that there was no solid evidence to suggest that these tumors were hormone-dependent $[3,5,6]$. They normally occur in younger age groups with the mean average age of 30-40 [4, 5]. Our patient, aged 35 was within the average age group, however was neither pregnant nor on hormonal treatment. She had no known risk factors or previous medical illness.

The clinical presentation of primary angiosarcomas can be varied; however, most present as a fast-growing palpable mass with overlying skin changes $[1,5,7]$. In our case, the initial history given was a gradual swelling of the left breast after a fall with trauma to the breast, leading the clinicians to suspect a hematoma, thus resulting in a wrong and delayed diagnosis. She also had bruises on other parts of the body, further confusing the diagnosis. Eventually after further probing, we managed to find out that the lump was actually palpable prior to the fall, which then pointed the diagnosis more towards a vascular tumor instead of purely a hematoma. Initial blood investigation showed thrombocytopenia (platelet reading was 66 with the unit of $10^{9} / \mathrm{L}$ ) and coagulopathy which was consistent with Kasabach-Merritt syndrome as reported by several journals [7].

The diagnosis of angiosarcomas can be challenging as most of them have non-specific radiological findings. On ultrasound, these tumors may present as hypo-, hyper-, or heterogeneous masses which are highly vascular in nature. Mammographically, the findings are very nonspecific with up to $33 \%$ of these tumors presenting as normal mammograms as reported by Liberman $[5,6,8]$. MRI is a more promising and supportive imaging modality which demonstrates low T1 signal and markedly high T2 signal. On dynamic phase, these tumors normally demonstrate rapid wash in followed by either prolonged enhancement or rapid wash out. In our case, the patient's ultrasound showed a large ill-defined heterogeneous mass with mixed solid and cystic components within, occupying almost the entire left breast. It had multiple large vessels within the mass. However, no significant left axillary lymphadenopathy was detected. Even though mammogram was not performed in this patient due to severe pain, an MRI scan was performed to further assess this mass. On MRI, the mass 
demonstrated predominantly low T1 signal, extremely high T2 signal with rapid wash in and prolonged enhancement on dynamic phase. Overall, even though the radiological findings from our patient's ultrasound and MRI may not have specific diagnostic features for angiosarcoma, it did help to characterize the mass, hence leading to the correct diagnosis.

There were journals which stated that fine-needle aspiration cytology and core biopsy are generally not helpful in the diagnosis of angiosarcoma as they give high false-negatives, as high as $37 \%[4,6,9]$. This did apply in our case, where the result of the core biopsy taken by the surgical team was inconclusive with blood clots seen as the mass was highly vascular and hemorrhagic, making diagnosis difficult. No strict contraindication for biopsy was previously mentioned by any journals, but it would be helpful for the biopsy to be done under ultrasound guidance in order to obtain proper samples and in avoiding the major vessels within the mass, hence reducing the difficulty in securing hemostasis, which happened in our case.

Histologically, angiosarcomas of the breast can be classified into grades 1,2 , and 3 or low, intermediate, and high. The cells are spindle shaped with large, oval nuclei, and vesicular chromatin. There are also large anastomosing vascular channels within. The high-grade tumors always demonstrated areas of 'blood lakes' which meant that there was bleeding into the surrounding stroma, as seen in our patient. Immunohistochemical staining-like factor VIII and CD31 plays an important role in differentiating angiosarcomas from other vascular tumors. Our patient's diagnosis was confirmed after proper microscopic examination together with immunohistochemical staining of the excised breast tissue.

The gold standard treatment for primary breast angiosarcomas is still surgery which includes total mastectomy or wide local excision, with or without axillary clearance. Total mastectomy is more favorable than wide local excision because of the resection margin status, as well as the high incidence of local recurrence in wide local excision as documented in most articles [3, 7]. Regional axillary clearance is not necessary because they tend to metastasize hematogenously, rather than lymphogenously [3, 4, 7, 9]. Our patient underwent total mastectomy with regional axillary clearance; however, she still developed local recurrence 6 months later.

The role of chemotherapy and radiotherapy in breast angiosarcomas is still not well established. However, some articles have reported that chemotherapy may be more effective in high grade tumors and in metastatic setting $[1,3-5,7,9]$. Few small sample studies also documented positive outcomes for both conservative surgery or post-mastectomy radiation [5, 9]. Most authors believe that both the chemotherapy and radiation therapy may reduce the rate of local recurrence and hence improve the total survival rate. Our patient received 6 cycles of chemotherapy with the drug Paclitaxel three weekly followed by adjuvant radiotherapy in the later part of her treatment.

Angiosarcomas of the breast are generally known to be poor in prognosis, especially for tumors more than 5 $\mathrm{cm}$ in size as well as for patients with distant metastasis at the time of diagnosis. A study done by Rosen et al. showed that the 5-year disease-free survival in high grade tumors was only $15 \%[1,5,6,9]$. Up to date, many debates are still going on regarding the prognostic factors and these include tumor size, surgical tumor margin status, and tumor grading. Our patient's tumors were more than $5 \mathrm{~cm}$ at the time of diagnosis with high tumor grading and these gave her poorer prognosis. Otherwise, no distant metastasis was seen. She was doing well in the first 6 months after surgery. Unfortunately, she returned with recurrence 6 months later.

\section{Conclusion}

Primary breast angiosarcomas are rare aggressive vascular tumors with generally poor prognosis. These tumors have no pathognomonic signs on radiological imaging, especially mammogram. However, ultrasound and MRI do help in characterizing these tumors and in leading to the correct diagnosis. There is no strict contraindication for biopsy of these tumors even though false negative value is high; nevertheless, it would be helpful to do it under ultrasound guidance to avoid unnecessary complications. A good clinical history in correlation with clinical and radiological findings are important to derive an early and precise diagnosis. In young patients with rapidly growing, highly vascular breast tumors, a differential diagnosis of primary angiosarcomas should always be considered until proven otherwise.

\section{Abbreviations}

MRI: Magnetic resonance imaging; CT: Computed tomography

\section{Acknowledgements \\ Dr Dhayal Balakrishnan, Hospital Kuala Lumpur provided some radiographic images.}

\section{Authors' contributions the manuscript. \\ Funding \\ N/A \\ Availability of data and materials \\ N/A}

Fui Tin Pang-main author in writing and compiling information. Sze Yuen Lee-helped in writing and grammar correction. MK-contributed in getting consent and pictures from the patient. All authors have read and approved 


\section{Declarations}

\section{Ethics approval and consent to participate}

Patient gave informed consent to use their data in case write up. No ethics approval is needed from the Hospital Tengku Ampuan Rahimah, Klang, for a case write up.

\section{Consent for publication}

Written informed consent was obtained from the patient for publication of this case report and accompanying images.

\section{Competing interests}

The authors declare that they have no competing interests.

Received: 7 January 2021 Accepted: 14 April 2021

Published online: 28 April 2021

\section{References}

1. Desbiens C, Hogue JC, Levesque Y (2011) Primary Breast Angiosarcoma: Avoiding a Common Trap. Case Reports Oncol Med 2011:5. Article ID 517047. https://doi.org/10.1155/2011/517047

2. Vorburger SA, Xing Y, Hunt KK, Lakin GE, Benjamin RS, Feig BW, Pisters PWT, Ballo MT, Chen L, Trent J III, Burgess M, Patel S, Pollock RE, Cormier JN (2005) Angiosarcoma of the breast. Cancer 104(12):2682-2688. https://doi. org/10.1002/cncr.21531

3. Bennani A, Chbani L, Lamchahab M, Wahbi M, Alaoui FF, Badioui I, Melhouf MA, Amarti A (2013) Primary angiosarcoma of the breast: a case report. Diagnostic Pathol 8(1):66. https://doi.org/10.1186/1746-1596-8-66

4. Rohan VS, Hanji AM, Patel JJ, Tankshali RA (2010) Primary angiosarcoma of the breast in a postmenopausal patient. J Cancer Res Ther 6(1):120-122. https://doi.org/10.4103/0973-1482.63543

5. Glazebrook KN, Magut MJ, Reynolds C (2008) Angiosarcoma of the breast. Am J Roentgenol 190(2):533-538. https://doi.org/10.2214/AJR.07.2909

6. Lim RF, Goei R (2007) Angiosarcoma of the breast. Radiographics 27(suppl_ 1):S125-S130

7. Bordoni D, Bolletta E, Falco G, Cadenelli P, Rocco N, Tessone A, Guarino S, Accurso A, Amato B, Magalotti C (2016) Primary angiosarcoma of the breast. Int J Surg Case Reports 20S(Suppl):12-15

8. Liberman L, Dershaw DD, Kaufman RJ, Rosen PP (1992) Angiosarcoma of the breast. Radiology 183(3):649-654. https://doi.org/10.1148/radiology.1 83.3.1584913

9. Pandey M, Martin MG (2014) Primary angiosarcoma of the breast: a case report and review of literature. World J Oncol 5(3):144-148. https://doi.org/1 $0.14740 /$ wjon 809 w

\section{Publisher's Note}

Springer Nature remains neutral with regard to jurisdictional claims in published maps and institutional affiliations.

\section{Submit your manuscript to a SpringerOpen ${ }^{\circ}$ journal and benefit from:}

- Convenient online submission

- Rigorous peer review

- Open access: articles freely available online

- High visibility within the field

- Retaining the copyright to your article 